Análisis territorial de las elasticidades de sustitución de los factores de producción en la industria manufacturera colombiana (1992-2018)

Julián Augusto Casas Herrera y Jhancarlos Gutiérrez Ayala 
Julián Augusto Casas Herrera y Jhancarlos Gutiérrez. Ayala

Análisis territorial de las elasticidades de sustitución de los factores de producción en la industria manufacturera colombiana $(1992-2018)$

Resumen: El articulo presenta la estimación de las elasticidades propias y de sustitución entre los insumos trabajo, materias primas, capital, gasto energético y otros gastos de producción para el sector industrial manufacturero de Colombia, la región central y el departamento de Boyacá. Las estimaciones se realizaron a través de la aplicación de funciones de costos translogarítmicas con datos para el periodo 1992 - 2018 de la Encuesta Anual Manufacturera (EAM). Dentro de los resultados se destaca el bajo nivel sustitutivo entre capital y trabajo en el escenario nacional, contando con una elasticidad de 0,08\%; en el caso del departamento la relación fue complementaria, aumentos de $1 \%$ en el precio del capital se relacionan con una reducción promedio de 0,17\% en la demanda de trabajo. Estos resultados muestran diferencias en la participación relativa promedio de los factores de producción en el costo total, lo que permite concluir que las dinámicas del sector industrial nacional, regional y del departamento son heterogéneas.

Palabras clave: Industria manufacturera; factores de producción; elasticidades; función translogaritmica; SURE.

Clasificación JEL: C51, D01, D24

\section{Territorial Analysis of the Elasticities of Substitution of Production Factors in the Colombian Manufacturing Industry (1992-2018)}

Abstract: The article presents the estimation of the price and substitution elasticities of demand between inputs, labor, intermediate material, capital, energy, and other production expenses for the industrial manufacturing sector of Colombia (the central region and the department of Boyaca). The estimates were made through the application of translogarithmic cost functions with data for the period 1992 - 2018 from the Annual Manufacturing Survey. Results show a low substitution level between capital and labor in the national scenario, with an elasticity of $0.08 \%$. In the case of the department of Boyaca, the relationship was complementary; increases of $1 \%$ in the price of capital are related to an average reduction of $0.17 \%$ in the demand for labor. These results show differences in the average relative participation of the production factors in the total cost, which allows for the conclusion that the dynamics of the national, regional, and department industrial sectors are heterogeneous.

Keywords: manufacturing industry; production factors; elasticities; translogarithmic function; SURE.

\section{(cc) BY-NC-SA}

Este artículo y sus anexos se distribuyen por la revista Lecturas de Economía bajo los términos de la Licencia Creative Commons Atribución-NoComercial-CompartirIgual 4.0. https://creativecommons.org/licenses/by-nc-sa/4.0/ 


\section{Analyse territoriale des élasticités de substitution des facteurs de production dans l'industrie manufacturière colombienne (1992 - 2018)}

Résumé: L'article présente l'estimation des élasticités propres et de substitution entre le travail, les matières premières, le capital, l'énergie et les autres dépenses de production pour le secteur de la fabrication industrielle en Colombie, dans la région centrale et le département de Boyacá. Les estimations ont été réalisées par l'application de fonctions de coût translogarithmiques avec des données pour la période 1992 - 2018 provenant de l'enquête annuelle sur les industries manufacturières. On distingue, parmi les résultats, le faible niveau de substitution entre capital et travail dans le scénario national, avec une élasticité de 0,08\%; dans le cas du département, la relation était complémentaire, des augmentations de $1 \%$ du prix du capital sont liées à une réduction moyenne de 0,17\% de la demande de travail. Ces résultats montrent des différences dans la participation relative moyenne des facteurs de production au coût total, ce qui permet de conclure que les dynamiques du secteur industriel national, régional et départemental sont hétérogènes.

Mots clés: Industrie manufacturière; facteurs de production; élasticités; fonction translogarithmique; SURE.

Cómo citar / How to cite this item:

Casas-Herrera, J. A. \& Gutiérrez-Ayala, J. (2021). Análisis territorial de las elasticidades de sustitución de los factores de producción en la industria manufacturera colombiana (1992-2018). Lecturas de Economía, 94, 233-265.

https://doi.org/10.17533/udea.le.n94a343836 


\title{
Análisis territorial de las elasticidades de sustitución de los factores de producción en la industria manufacturera colombiana (1992-2018)
}

\author{
Julián Augusto Casas Herrera $\odot^{\mathrm{a}}$ y Jhancarlos Gutiérrez Ayala $\odot^{\mathrm{b}}$
}

-Introducción. -I. Antecedentes. -II. Modelo econométrico -III. Datos y variables -IV. Estimación y discusión de los resultados. -Conclusiones. -Referencias.

Primera versión recibida el 17 de septiembre de 2020; versión final aceptada el 02 de noviembre de 2020

\section{Introducción}

Durante el periodo 1992-2018 la industria manufacturera colombiana representó en promedio un 13,7 \% del Producto Interno Bruto (PIB), según datos del Departamento Administrativo Nacional de Estadística (DANE); en contraste con el sector primario $(15,3 \%)$ y el sector servicios $(50 \%)$. Además, la participación del sector industrial en el PIB pasó del 18,1\% al 10,5\% entre 1992 y 2018. El motivo de la reducción en su participación puede deberse a su tasa de crecimiento promedio (1,3\%), la cual es inferior a la del sector primario $(2,4 \%)$ y el sector servicios $(4,29 \%)$, los cuales ganaron participación en el PIB durante el mismo periodo.

Según Amézquita (2008) la composición de la estructura industrial colombiana no se ha modificado drásticamente desde 1970, la producción se ha concentrado en la elaboración de alimentos y bebidas, productos de la refinación de petróleo, productos de madera y metal (los cuales son intensivos en el uso de recursos naturales); textiles, confecciones, artes gráficas, cuero,

a Julián Augusto Casas Herrera: docente de economía de la Universidad Pedagógica y Tecnológica de Colombia, Tunja, Colombia Dirección electrónica: julian.casas01@uptc.edu.co https://orcid.org/0000-0001-6606-5059

b Jhancarlos Gutiérrez. Ayala: investigador de Universidad Pedagógica y Tecnológica de Colombia. Tunja. Colombia Dirección electrónica: jhancarlos.gutierrez@uptc.edu.co https://orcid.org/0000-0002-4756-7683 
calzado y marroquinería (intensivos en mano de obra); industrias químicas y plásticos, maquinaria y equipos (intensivos en conocimiento y tecnología).

Es precisamente el uso intensivo de factores de producción un punto clave en el desempeño del sector industrial, porque el uso óptimo de estos le permite al productor maximizar beneficios y minimizar costos. Por tanto, una elección adecuada de los insumos de producción (mano de obra, capital, materias primas, energía eléctrica u otros) coadyuvarán a lograr mejores resultados para los intereses de las empresas, así como de la industria.

En ese sentido, cuando se realiza una revisión de la literatura referente al sector industrial, se encuentran análisis desde la perspectiva de las características de la inversión, los efectos por la firma de acuerdos internacionales y condiciones referentes a su crecimiento. Estos análisis cuentan con altos niveles de validez cuando se pretende observar las características generales de las empresas que pertenecen al sector industrial. Sin embargo, vale la pena preguntarse si el comportamiento industrial en distintos niveles territoriales es el mismo, o si existen condiciones diferenciales que caractericen a las empresas de un territorio frente a las de otros niveles.

En este escenario, se considera válido realizar un estudio que haga un diagnóstico relacionado con las dinámicas de demanda de factores de producción para tres niveles territoriales: nacional, regional ${ }^{1}$ y el departamento de Boyacá. La inclusión de este último responde a la ausencia de trabajos relacionados para la industria departamental, la cual carece de estudios recientes que permitan entender la dinámica del territorio frente a estándares nacionales ${ }^{2}$. Al considerar tres niveles territoriales se pretende observar los comportamientos particulares de las empresas, identificando diferencias en el uso de factores y, por ende, de las condiciones de optimización de la producción; esto bajo la premisa de que las condiciones sociales, políticas, económicas o ambientales de una región pueden generar cambios en su estructura productiva.

1 Se toman datos agregados para las empresas manufactureras de los departamentos de Cundinamarca, Santander y Boyacá, que conjuntamente representaron, en promedio, para el periodo 1992-2018 un 12,6\% del PIB y un $11 \%$ de la producción industrial.

2 Para otra aproximación regional ver Ávila, Camargo, y Marques (2019). 
El artículo se divide en cinco secciones, aparte de esta introducción. En la primera se incluyen aspectos teóricos referentes a la metodología, junto con la importancia de los análisis de la demanda de factores de producción y las elasticidades de sustitución. La segunda plantea un modelo basado en una función de costos translogarítmica y la estructura del sistema de ecuaciones a estimar. La tercera referencia los datos utilizados de la Encuesta Anual Manufacturera (EAM) durante el periodo de estudio. La cuarta presenta el análisis de los resultados de la estimación de elasticidades de sustitución de seis factores de producción ${ }^{3}$ para la industria manufacturera nacional, regional y departamental. Finalmente, se presentan las conclusiones.

\section{Antecedentes}

Este documento estima las elasticidades propias y cruzadas de demanda de los factores de producción en la industria manufacturera colombiana, regional y del departamento de Boyacá, con datos de la Encuesta Anual Manufacturera (EAM) del DANE para el periodo comprendido entre 1992 y 2018, mediante la aplicación de funciones de costos translogarítmicas considerando al trabajo, materias primas, capital, gasto energético y otros costos de producción (relacionados principalmente con gastos financieros) como factores de producción del sector. Esto con el objetivo de realizar comparaciones y encontrar diferencias en el comportamiento de la industria en distintos niveles.

El estudio de la relación entre el uso de factores y el nivel de producción de una empresa o industria adquiere importancia al considerar las condiciones que llevan al empresario a tratar de elegir la asignación óptima de los insumos para conseguir el mayor nivel de producción posible, a un mínimo costo (Le, 2019). Esta relación se manifiesta a través de la función de producción ya que, según Berndt y Christensen (1973), Aigner, Lovell y Schmidt (1977), Berndt y Wood (1987) y Carro y González (2019), esta permite identificar los recursos empleados en el proceso de producción de bienes y servicios de una economía.

La función de producción no solo se emplea para el agregado de la economía, sino que también es la herramienta que permite observar cómo

3 Trabajo, mano de obra, materias primas, capital, energía eléctrica y otros factores de producción. 
Casas Herrera y Gutiérrez Ayala: Análisis territorial de las elasticidades de sustitución...

está estructurado el proceso de producción de una empresa (Cepas y Dios, 1999). Valga decir que la función de producción recoge dos tipos de relaciones. Por una parte, relaciona el uso de insumos con el nivel de producción lo que determina la existencia de rendimientos a escala (crecientes, constantes o decrecientes). Por otra, permite establecer la relación interna entre factores de producción, a través de las elasticidades de sustitución entre estos, que le muestran al productor la capacidad de maniobrabilidad que tiene en torno de la elección de determinados niveles de insumos para tener un nivel deseado de producción.

Según Zha y Zhou (2014), las elasticidades parciales de sustitución se introducen a partir del trabajo realizado por Allen (1938) y Uzawa (1962) quienes analizan el cambio en la demanda de dos factores $i$ y $j$ ante el comportamiento de los precios de estos. Estas elasticidades permiten determinar el grado en el que la demanda de un factor $i$ responde a cambios del precio del factor $j$, definiéndose como una elasticidad cruzada precio de la demanda.

Además, según Hisnanick y Kyer (1995) las elasticidades Allen-Uzawa permiten clasificar la relación entre dos factores, ya sean sustitutos (un aumento del precio del factor $j$ genera aumentos en la demanda del factor $i$ respectivamente), complementarios (un aumento del precio del factor $j$ genera reducciones en la demanda del factor $i$ respectivamente) o independientes (un aumento del precio del factor $j$ no afecta la demanda del factor $i$ ). Estas elasticidades han sido utilizadas en diversos trabajos que parten de las formas funcionales planteadas por Cobb y Douglas (1928), Diewert (1971) y Berndt y Christensen (1973).

La forma funcional más usada, en estudios empíricos de la elasticidad, es la de Cobb y Douglas (1928). Sin embargo, esta no ha escapado a la crítica dadas las condiciones de separabilidad ${ }^{4}$ impuestas, las cuales determinan que los factores de producción pueden verse como independientes. Para

4 "La separabilidad de factores representa la manera en que la tasa marginal de sustitución técnica entre dos factores responde a cambios en la cantidad de otro factor de producción. Por ejemplo, si el aumento en el acervo de capital hace que los productos marginales de la mano de obra y los materiales se desplacen en la misma proporción, entonces se dice que la mano de obra y los materiales son separables del capital” (Ibarra \& García, 2013, pp. 157-158). 
ejemplificar esta condición, siguiendo los planteamientos de Álvarez, Arias y Orea (2003) e Ibarra y García (2016) piense en una empresa que usa tres factores de producción: capital, trabajo y materias primas. La restricción de separabilidad de la función Cobb-Douglas implica que cualquier cambio en el acervo de capital causará el mismo efecto sobre los factores restantes, esto es que la elasticidad parcial de sustitución entre capital y trabajo es la misma que la elasticidad entre capital y materias primas lo cual se puede interpretar como un sesgo de análisis sectorial.

Ante la limitación de la función original de Cobb-Douglas, Berndt y Christensen (1973) y Christensen, Jorgenson y Lau (1973) propusieron la función de producción logarítmica trascendental-translogarítmica, que surgió de combinar las funciones de Cobb-Douglas (1928) con la generalizada de Leontief, desarrollada por Diewert (1971). Como se muestra en la ecuación 1, la forma funcional de la función de producción translogarítmica (FPT), además de tener términos lineales y cuadráticos, permite la inclusión de insumos sin imponer condiciones de separabilidad de factores a priori (Ibarra y García, 2013).

$$
\begin{aligned}
\ln (Y)= & \ln \alpha_{0}+\alpha_{A} \ln (A)+\sum_{i=1}^{n} \alpha_{i} \ln \left(X_{i}\right)+\frac{1}{2} \gamma_{A} \ln (A)^{2} \\
& +\frac{1}{2} \sum_{i=1}^{n} \sum_{j=1}^{n} \gamma_{i j} \ln \left(X_{i}\right) \ln \left(X_{j}\right)+\sum_{i=1}^{n} \gamma_{i A} \ln \left(X_{i}\right) \ln (A),
\end{aligned}
$$

donde, $Y$ representa la producción, $A$ es el factor tecnológico, $X_{i}$ y $X_{j}$ son los factores de producción (trabajo, materias primas, capital y energía eléctrica), $\alpha$ y $\gamma$ son parámetros que relacionan la tecnología y los factores. Esta forma funcional permite evaluar el cumplimiento de las condiciones de separabilidad, la existencia de rendimientos constantes a escala y otras condiciones asociadas a las elasticidades Allen-Uzawa.

A partir de la FPT es posible construir una función de costos translogarítmica (FCT) dada la dualidad del productor, que considera su comportamiento como maximizador del ingreso o minimizador de los costos. La construcción de la FCT es útil en el campo de la investigación ya que, como 
Casas Herrera y Gutiérrez Ayala: Análisis territorial de las elasticidades de sustitución...

se muestra en la ecuación 2, esta permite establecer relaciones basadas en los índices de precios de los factores de producción y , además de las elasticidades Allen-Uzawa, la participación histórica de los factores junto con la evaluación del comportamiento de los costos industriales a nivel empresarial o agregado.

$$
\begin{aligned}
\ln (C)= & \ln \left(\alpha_{0}\right)+\sum_{i=1}^{n} \alpha_{i} \ln \left(P_{i}\right)+\frac{1}{2} \sum_{i=1}^{n} \sum_{j=1}^{n} \gamma_{i j} \ln \left(P_{i}\right) \ln \left(P_{j}\right) \\
& +\alpha_{Y} \ln (Y)+\frac{1}{2} \gamma_{Y}(\ln (Y))^{2}+\sum_{i=1}^{n} \gamma_{i Y} \ln \left(P_{i}\right) \ln (Y)
\end{aligned}
$$

donde, $\ln (C)$ es el logaritmo del costo total de la producción, $\ln (Y)$ es el logaritmo de la producción, $\ln (P i)$ y $\ln (P j)$ representan el logaritmo de los precios de los factores de producción $i$ y $j$. Según la Clemson University (2005) la FCT representa las condiciones de minimización de costos de una firma o industria, a partir de la relación entre los precios y la demanda de los factores de producción. Para mostrar la versatilidad de la FPT y la FCT se presentan algunos resultados obtenidos en distintos documentos que las utilizaron.

Salgado y Bernal (2010) emplean la FPT para estimar elasticidades precio y sustitución de la demanda por insumos (capital, trabajo, energía y transporte) para la industria manufacturera mexicana para el periodo 1996-2003. Los autores encontraron una relación sustitutiva entre el capital y la mano de obra, caracterizando que un aumento de un $1 \%$ en el índice de precios del capital, provocó en promedio para su periodo de análisis un aumento de $0,5 \%$ en la demanda de mano de obra.

Ibarra y García (2013) realizan, para la industria maquiladora mexicana, una evaluación de los supuestos de rendimientos constantes a escala y separabilidad entre factores productivos, a través de la estimación de una FCT para el periodo 1990-2006. Los autores encuentran que la industria presentó rendimientos decrecientes a escala. Esto es que la generación de una unidad extra de producto aumenta su costo frente a la última unidad producida. Adicionalmente, encuentran que mediante el uso de la FCT se rompen los supuestos de separabilidad entre los factores de producción, condición que 
contradice los supuestos con los que se trabaja a priori en el caso de usar otras formas funcionales como la Cobb-Douglas. Por último, evidencian que en la industria maquiladora los factores trabajo, capital y materias primas son sustitutivos entre sí.

Para la industria manufacturera peruana, Cabezas (1997) realiza un estudio de las posibilidades de sustitución entre los insumos capital, trabajo, energía y materias primas para el periodo 1974 a 1992, estimando las elasticidades AllenUzawa para diferentes actividades de la industria. Los resultados mostraron relaciones de complementariedad entre el trabajo y el capital para actividades como alimentos, textiles, productos de madera, papel, maquinaria y minerales no metálicos; mientras que para "otras industrias manufactureras", dentro de las que se incluyen la producción de joyería, juguetes y artículos de oficina, se observó una relación de sustitución entre los mismos factores.

Kuan, Hongchang, Yuxin, Jefferson y Rawski (1988) emplearon funciones de producción Cobb-Douglas y translogarítmicas para calcular la productividad de factores para la industria China en el periodo 1953-1985. Sus estimaciones mostraron que el aumento en un $1 \%$ del stock de capital generó, en promedio, un incremento entre $0,44 \%$ y $0,63 \%$. Además, los autores evidenciaron cambio estructural de la relación de productividad de los factores de producción, puesto que la productividad entre 1957-1978 fue de $1,1 \%$ y entre 1978-1985 fue de 2,7\%, lo que podría ser un argumento a favor de la reforma económica aplicada en 1978, que impactó en el crecimiento industrial de China.

Looi Kee, Nicita y Olarreaga (2008) analizaron el comportamiento de las importaciones de 117 países durante el periodo 1988-2001, a través de una FPT. Esta función, consideró como variable clave la firma de tratados de libre comercio, el rol del trabajo, el capital y la tierra agrícola. Los resultados mostraron una elasticidad negativa del precio-propio de las importaciones de $-3,12 \%$. Esto es que un aumento de un $1 \%$ del precio de las importaciones generado por la firma de acuerdos comerciales o por factores macroeconómicos propios de la nación — tales como la variación de la tasa de cambio- causó reducciones del 3,12\% en las importaciones, encontrando un alto grado de sensibilidad en la demanda. 
Casas Herrera y Gutiérrez Ayala: Análisis territorial de las elasticidades de sustitución...

Saal y Parker (2000) estiman una FCT ${ }^{5}$, para Inglaterra y Gales para el periodo 1985 a 1999, con el fin de analizar el comportamiento de los costos generados tras la privatización del servicio de acueducto en estos países. Los resultados encontrados muestran que el comportamiento de los costos de purificación del agua tras la privatización aumentó de forma constante. El aumento de costos se presentó debido al uso intensivo de capital en reemplazo de la mano de obra. Adicionalmente, encontraron que el uso de funciones tipo Cobb-Douglas es ineficiente para el tratamiento de los costos dado el no cumplimiento del supuesto de separabilidad de factores.

En Colombia, el estudio de Ramírez (2008), analizó la participación del trabajo (calificado y no calificado) y del capital en la industria manufacturera para el periodo 1994-2005. El autor halló "que el trabajo calificado ha ganado participación al interior del proceso productivo en detrimento del trabajo no calificado y el capital" (Ramírez, 2008, p. 42). Además, indica que los precios de los tres insumos aumentaron constantemente, lo que permite catalogar al trabajo calificado como un bien de lujo cuya demanda aumenta a pesar del incremento de su precio. Finalmente, encontró una relación de complementariedad entre el trabajo calificado y el capital, de modo que el aumento del salario causó reducciones en la demanda de capital y viceversa ${ }^{6}$.

\section{Modelo econométrico}

A partir de los elementos teóricos esbozados en la sección anterior, que mostró la versatilidad que tiene la FPT, se procede a plantear el modelo para estimar la participación y las elasticidades de sustitución entre los insumos trabajo, materias primas, capital, gasto energético y otros gastos de producción para el sector industrial manufacturero de Colombia, la región y del departamento de Boyacá.

5 Los autores califican esta función como "superior a otras empleadas ya que es mejor cuando se pretende distinguir diferentes causas del cambio en los costos" (Saal \& Parker, 2000, p. 264).

6 A una similar conclusión llegan Ávarez Mendoza y Defaz Novillo (2019) para el caso de la industria manufacturera ecuatoriana. 
Para el propósito que persigue este documento, se optó usar las FPT planteadas por Berndt y Christensen (1973) y Andrade, Gaspar, y Bittencourt (2014), las cuales están expresadas en la ecuación 1.

Considerando que los productores buscan maximizar beneficios y minimizar costos, se puede "modelar una tecnología de producción directamente de una función de producción o de una función de costos" (Cabezas, 1997, p. 515). En consecuencia, se eligió usar la FCT como instrumento de análisis de las elasticidades de sustitución entre factores. Los factores que se tienen en cuenta en este documento son: trabajo $(L)$, materias primas $(M)$, capital $(K)$, energía eléctrica $(E)$ y otros factores de producción $(O) . \mathrm{Al}$ incorporar estos factores en la ecuación 2 , se obtiene la ecuación 3 :

$$
\begin{aligned}
\ln (C)= & \ln \left(\alpha_{0}\right)+\alpha_{L} \ln \left(P_{L}\right)+\alpha_{M} \ln \left(P_{M}\right)+\alpha_{K} \ln \left(P_{K}\right)+\alpha_{E} \ln \left(P_{E}\right) \\
& +\alpha_{O} \ln \left(P_{O}\right)+\gamma_{L M} \ln \left(P_{L}\right) \ln \left(P_{M}\right)+\gamma_{L K} \ln \left(P_{L}\right) \ln \left(P_{K}\right) \\
& +\gamma_{L E} \ln \left(P_{L}\right) \ln \left(P_{E}\right)+\gamma_{L O} \ln \left(P_{L}\right) \ln \left(P_{O}\right) \\
& +\gamma_{M K} \ln \left(P_{M}\right) \ln \left(P_{K}\right)+\gamma_{M E} \ln \left(P_{M}\right) \ln \left(P_{E}\right) \\
& +\gamma_{M O} \ln \left(P_{M}\right) \ln \left(P_{O}\right)+\gamma_{K E} \ln \left(P_{K}\right) \ln \left(P_{E}\right) \\
& +\gamma_{K O} \ln \left(P_{K}\right) \ln \left(P_{O}\right)+\gamma_{E O} \ln \left(P_{E}\right) \ln \left(P_{O}\right) \\
& +\frac{1}{2} \gamma_{L L}\left(\ln \left(P_{L}\right)\right)^{2}+\frac{1}{2} \gamma_{M M}\left(\ln \left(P_{M}\right)\right)^{2}+\frac{1}{2} \gamma_{K K}\left(\ln \left(P_{K}\right)\right)^{2} \\
& +\frac{1}{2} \gamma_{E E}\left(\ln \left(P_{E}\right)\right)^{2}+\frac{1}{2} \gamma_{O O}\left(\ln \left(P_{O}\right)\right)^{2}+\alpha_{Y} \ln (Y) \\
& +\frac{1}{2} \gamma_{Y Y}(\ln (Y))^{2}+\gamma_{Y L} \ln \left(P_{L}\right) \ln (Y) \\
& +\gamma_{Y M} \ln \left(P_{M}\right) \ln (Y)+\gamma_{Y K} \ln \left(P_{K}\right) \ln (Y) \\
& +\gamma_{Y E} \ln \left(P_{E}\right) \ln (Y)+\gamma_{Y O} \ln \left(P_{O}\right) \ln (Y)
\end{aligned}
$$

donde, $(\ln C)$ relaciona el nivel de costos con el uso de cinco insumos agregados: trabajo $\left(P_{L}\right)$, materias primas $\left(P_{M}\right)$, capital $\left(P_{K}\right)$, servicios públicos $\left(P_{E}\right)$ y otros costos asociados a la producción $\left(P_{O}\right)$.

Según Berndt y Christensen (1973) y Cabezas (1997) existen algunos supuestos que deben cumplirse de la ecuación 3, para asegurar que esta se 
Casas Herrera y Gutiérrez Ayala: Análisis territorial de las elasticidades de sustitución...

comporte adecuadamente ${ }^{7}$. Para este trabajo se plantean dos. Por un lado, la FCT debe ser homogénea de grado uno en precios; es decir, dado un nivel de producción fija, un cambio en el precio de los factores de producción debe verse reflejado en un aumento de igual proporción en el costo total (esta condición se presenta con las ecuaciones $4,5,6$ ).

$$
\begin{gathered}
\sum_{i=1}^{n} \alpha_{i}=1 \\
\sum_{i=1}^{n} \gamma_{i Y}=0 \\
\sum_{i=1}^{n} \gamma_{i j}=\sum_{i=1}^{n} \gamma_{j i}=0 .
\end{gathered}
$$

Por otro lado, se debe cumplir la restricción de simetría en los coeficientes. De forma que la relación entre el precio del insumo i respecto del insumo $j$ es la misma relación existente entre $j$ e $i$. Esta condición se define como:

$$
\gamma_{i j}=\gamma_{j i}
$$

Dado que la ecuación 3 representa el nivel de precios que minimiza los costos de producción (se supone que se está en un mercado de factores competitivo), al aplicar el Lema de Shepard es posible obtener las funciones de demanda no condicionada para cada uno de los factores de producción. De esta forma, se construye un sistema de ecuaciones que representan la

7 Se plantea un comportamiento "adecuado" de la función siempre que esta cumpla con las condiciones resumidas por la Clemson University (2005): 1. La demanda de insumos tiene pendiente negativa. 2. Los efectos de los precios cruzados son simétricos. 3. La suma de las elasticidades de precio propia y cruzada es igual a cero. 4. Un aumento proporcional en el precio de todos los insumos debe desplazar el costo en la misma cantidad manteniendo constante la producción. 
demanda de factores de producción en la industria de los cinco insumos (ecuación 8).

$$
\begin{aligned}
S_{L}= & \alpha_{L}+\gamma_{L L} \ln \left(P_{L}\right)+\gamma_{L M} \ln \left(P_{M}\right)+\gamma_{L K} \ln \left(P_{K}\right)+\gamma_{L E} \ln \left(P_{E}\right) \\
& +\gamma_{L O} \ln \left(P_{O}\right)+\gamma_{L Y} \ln (Y)+\mu \\
S_{M}= & \alpha_{M}+\gamma_{L M} \ln \left(P_{L}\right)+\gamma_{M M} \ln \left(P_{M}\right)+\gamma_{M K} \ln \left(P_{K}\right) \\
& +\gamma_{M E} \ln \left(P_{E}\right)+\gamma_{M O} \ln \left(P_{O}\right)+\gamma_{M Y} \ln (Y)+v \\
S_{K}= & \alpha_{K}+\gamma_{K L} \ln \left(P_{L}\right)+\gamma_{K M} \ln \left(P_{M}\right)+\gamma_{K K} \ln \left(P_{K}\right) \\
& +\gamma_{K E} \ln \left(P_{E}\right)+\gamma_{K O} \ln \left(P_{O}\right)+\gamma_{K Y} \ln (Y)+z \\
S_{E}= & \alpha_{E}+\gamma_{L E} \ln \left(P_{L}\right)+\gamma_{E M} \ln \left(P_{M}\right)+\gamma_{E K} \ln \left(P_{K}\right) \\
& +\gamma_{E E} \ln \left(P_{E}\right)+\gamma_{E O} \ln \left(P_{O}\right)+\gamma_{E Y} \ln (Y)+u \\
S_{O}= & \alpha_{O}+\gamma_{L O} \ln \left(P_{L}\right)+\gamma_{O M} \ln \left(P_{M}\right)+\gamma_{O K} \ln \left(P_{K}\right) \\
& +\gamma_{O E} \ln \left(P_{E}\right)+\gamma_{O O} \ln \left(P_{O}\right)+\gamma_{O Y} \ln (Y)+\omega,
\end{aligned}
$$

donde $S_{L}+S_{M}+S_{K}+S_{E}+S_{O}=1$.

Hasta este punto se ha construido un sistema de cinco ecuaciones que representan la demanda de los insumos $(L, M, K, E, O)$. Además, se ha propuesto que este sistema debe cumplir con las condiciones de homogeneidad y simetría planteadas en las ecuaciones 4, 5, 6 y 7. Con el objetivo de verificar el cumplimiento de estas condiciones se realiza una estimación de la ecuación (o sistema) 8, mediante mínimos cuadrados ordinarios (MCO).

La estimación por MCO, tradicionalmente, se caracteriza por permitir la obtención de los mejores estimadores lineales insesgados (MELI). Sin embargo, para el caso del sistema 8 se encuentra un problema de multicolinealidad casi perfecta, esto debido a que las variables exógenas de las cinco ecuaciones son las mismas. Igualmente, el sistema plantea una interrelación entre índices de precios y demandas de insumos, por lo que hay una múltiple dependencia entre variables y ecuaciones. La existencia de multicolinealidad, causa que "los coeficientes de regresión, aunque sean determinados, posean grandes errores estándar (...) lo cual significa que los coeficientes no pueden ser estimados con gran precisión o exactitud" (Gujarati y Porter, 2010, p. 323). 
Casas Herrera y Gutiérrez Ayala: Análisis territorial de las elasticidades de sustitución...

Debido a la existencia de multicolinealidad en el sistema, se debe recurrir a otra metodología de estimación. Para el caso de análisis de curvas de demanda de productos y de insumos en la economía, Rossi (1985) propone el uso de la metodología de ecuaciones aparentemente no relacionadas (SURE) ${ }^{8}$, que permite la estimación de un sistema de ecuaciones que se relacionan entre sí, ya sea por una variable o por sus residuos. Según Baltagi (2006) la metodología SURE se emplea para estimar modelos que explican no solo la variación de una variable endógena, sino también la variación de un conjunto $n$ de variables.

La metodología SURE fue desarrollada por Zellner (1962), a partir de la estimación de mínimos cuadrados generalizados (MCG) en tres etapas como respuesta a la ineficiencia de los estimadores de MCO. Esta metodología permite la corrección de la multicolinealidad entre las variables exógenas de un sistema de ecuaciones. Finalmente, Geweke (2005 p. 162) afirma que SURE

es el modelo econométrico más utilizado después de las regresiones lineales. La razón es que proporciona una representación simple y útil de los sistemas de ecuaciones de demanda que surgen en las teorías estáticas neoclásicas del comportamiento del productor y del consumidor.

Con el propósito de estimar el sistema 8, y considerando que la sumatoria de la participación de los factores de producción es igual a uno, Rossi (1985) propone que es posible eliminar una de las ecuaciones del sistema y emplearla como condición de restricción. De esta forma, es posible aplicar la metodología SURE eliminando las condiciones de multicolinealidad del sistema. En este caso, se suprime la ecuación correspondiente a otros factores de producción. Por tanto, el sistema 8 en su forma reducida queda de la siguiente manera:

$$
S_{i}=\alpha_{i}+\sum_{j}^{n} \gamma_{i j} \ln \left(\frac{P_{j}}{P_{O}}\right)+\gamma_{i Y} \ln (Y)
$$

8 Seemingly Unrelated Regression Estimator por sus siglas en inglés. 
siendo $i, j$ los factores de producción (trabajo, materias primas, capital y energía eléctrica), $\left(S_{i}\right)$ representa la participación de cada uno de los factores en el costo total $\left(\ln \left(\frac{P_{i}}{P_{O}}\right)\right)$. Es la relación entre el logaritmo de los índices de precios de los factores respecto del logaritmo de los precios de otros factores de producción. Esta última relación actúa como la condición de restricción, que garantiza el cumplimiento de los supuestos (homogeneidad de grado uno y simetría) y permite la estimación de la ecuación 9 mediante SURE.

La estimación del sistema conformado por las funciones de demanda de los factores de producción permite emplear los estimadores para el cálculo de elasticidades cruzadas de la demanda entre insumos. Siguiendo a Mundlak (1968) la estimación de las elasticidades de sustitución parcial de Allen-Uzawa, que definen la respuesta de la demanda de un factor $i$ frente al aumento del precio del factor $j\left(\sigma_{i j}\right)$, para la FCT se puede obtener del siguiente modo:

$$
\sigma_{i j}=\sigma_{j i}=\frac{\widehat{\gamma}_{i j}+\bar{S}_{i} S_{j}}{\bar{S}_{i} \bar{S}_{j}} .
$$

Además, es posible estimar las elasticidades precio-propias de la demanda de insumos de producción así:

$$
\sigma_{i i}=\frac{\widehat{\gamma}_{i i}+\bar{S}_{i}^{2} S_{j}}{\bar{S}_{i}^{2}}
$$

Antes de proceder a la estimación de la ecuación 9, se presenta una descripción de los datos y variables empleadas.

\section{Datos y variables}

Los datos empleados en este documento fueron tomados de la Encuesta Anual Manufacturera (EAM), aplicada por el DANE, la cual proporciona información referente a producción, empleo, inversión, transporte y otras variables relacionadas con el desempeño de las empresas industriales con más de 10 empleados a nivel nacional (DANE, 2019). 
Casas Herrera y Gutiérrez Ayala: Análisis territorial de las elasticidades de sustitución...

De forma específica se emplean series históricas territoriales desde el año $1992^{9}$ hasta el 2018 de las variables producción, salario de los trabajadores, costo de las materias primas empleadas, costo de capital, costo de la energía eléctrica y otros costos asociados a la producción. A continuación, se muestra la definición de estas variables de acuerdo con la metodología del DANE ${ }^{10}$.

Producción (Y): la serie de valor de los productos y subproductos manufacturados en el año "corresponde al valor de todos los productos manufacturados en el establecimiento y destinados a la venta" (DANE, 2019, p. 9). Esta variable considera la producción de todos los productos sin importar si son vendidos o no, incluyendo la variación de inventarios.

Salario de los trabajadores (L): esta variable incluye dos aspectos. Por un lado, se considera el costo de contratación, pago de salarios y prestaciones de los empleados permanentes. Por otro lado, incluye "el valor causado por la contratación esporádica, ocasional o accidental de personas naturales o jurídicas, para la prestación de servicios profesionales o técnicos al proceso productivo del establecimiento" (DANE, 2019, p. 9).

Costo de materias primas (M): corresponde al costo de las materias primas, insumos, empaques y materiales empleados en el proceso de producción. Se consideran todos los insumos empleados en la manufactura, sin importar si hacen parte o no de la presentación final del producto.

Costo de capital (K): considera el pago de intereses financieros, el arriendo y mantenimiento de establecimientos físicos como locales y plantas de producción ${ }^{11}$, así como la compra, arrendamiento y mantenimiento de las maquinarias empleadas en el proceso de manufactura ${ }^{12}$.

Costo de la energía eléctrica (E): es el valor pagado por los kilovatios (kWh) consumidos durante el proceso de producción.

\footnotetext{
9 A partir de este año los microdatos son publicados por el DANE.

10 Para ver recientes estudios se sugiere ver Vidal (2020).

11 Sin considerar el costo de servicios públicos.

12 Se incluyen también los costos de transporte y aseguramiento de la maquinaria.
} 
Otros costos de producción (O): recoge los demás costos en que incurre la empresa en el proceso de producción, tales como comisiones por ventas, fletes, acarreos, papelería, entre otros.

Aunque desde 1992 hasta el 2018, la metodología de la EAM se ha modificado cuatro veces ${ }^{13}$, las variables empleadas en el documento no sufrieron modificaciones. Ya que el objetivo de este artículo es analizar la relación entre la demanda y el gasto en la compra de factores de producción, es pertinente observar el comportamiento del gasto en insumos, para identificar diferencias en el comportamiento en los tres niveles territoriales analizados.

La Figura 1 muestra la variación porcentual del gasto medio en cada factor de producción para la industria manufacturera de Colombia, la región ${ }^{14}$ y el departamento de Boyacá. Los paneles a-e presentan altos niveles de volatilidad, principalmente las series referentes al departamento de Boyacá, por lo que se procede a identificar las principales diferencias entre el comportamiento del gasto en la industria del departamento frente a la industria manufacturera regional y nacional.

La tasa de variación promedio del gasto en trabajo para la industria manufacturera nacional es de 3,94\%, nivel de crecimiento similar al de la región (3,98\%). Sin embargo, en el caso de las empresas de Boyacá, el ritmo de crecimiento es menor con una variación promedio de 1,44\%. Esto se explica por la convergencia que han tenido las empresas nacionales y de la región para acercarse al nivel de salarios del departamento. Puesto que el salario mensual promedio de Boyacá para el 2018 fue de $\$ 1994$ 791, mientras que para Colombia y la región fueron de $\$ 1480640$ y $\$ 1530530$ respectivamente.

El panel b muestra la variación del gasto en materias primas. Se observa un alto grado de volatilidad principalmente en la región y en Boyacá. La tasa media de variación para la industria manufacturera fue de 4,98\%. Sin embargo, se encuentra que el aumento del gasto en materias primas de los empresarios de la región está por encima del promedio nacional (8,49\%). Para el caso de Boyacá se encuentra un ritmo de crecimiento del gasto más lento $(3,56 \%)$.

$131995,2000,2008$ y 2015 .

14 Conformada por los departamentos de Santander, Cundinamarca y Boyacá. 
Casas Herrera y Gutiérrez Ayala: Análisis territorial de las elasticidades de sustitución...

Figura 1. Variación porcentual media del gasto en factores (1992-2018)

(a) Gasto en trabajo

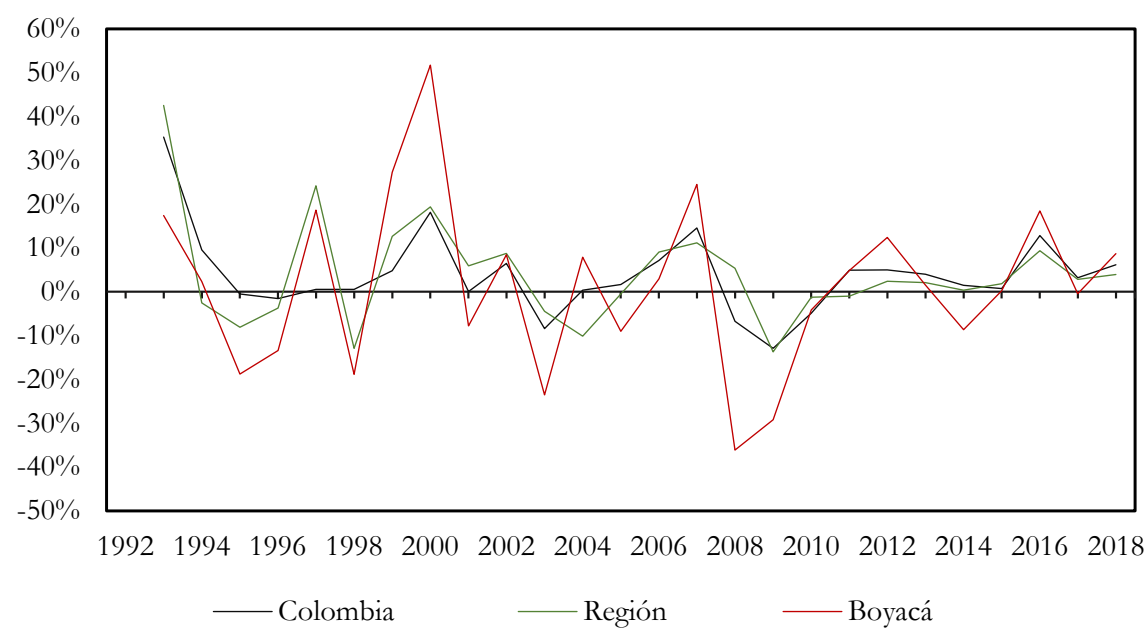

(b) Gasto en materias primas

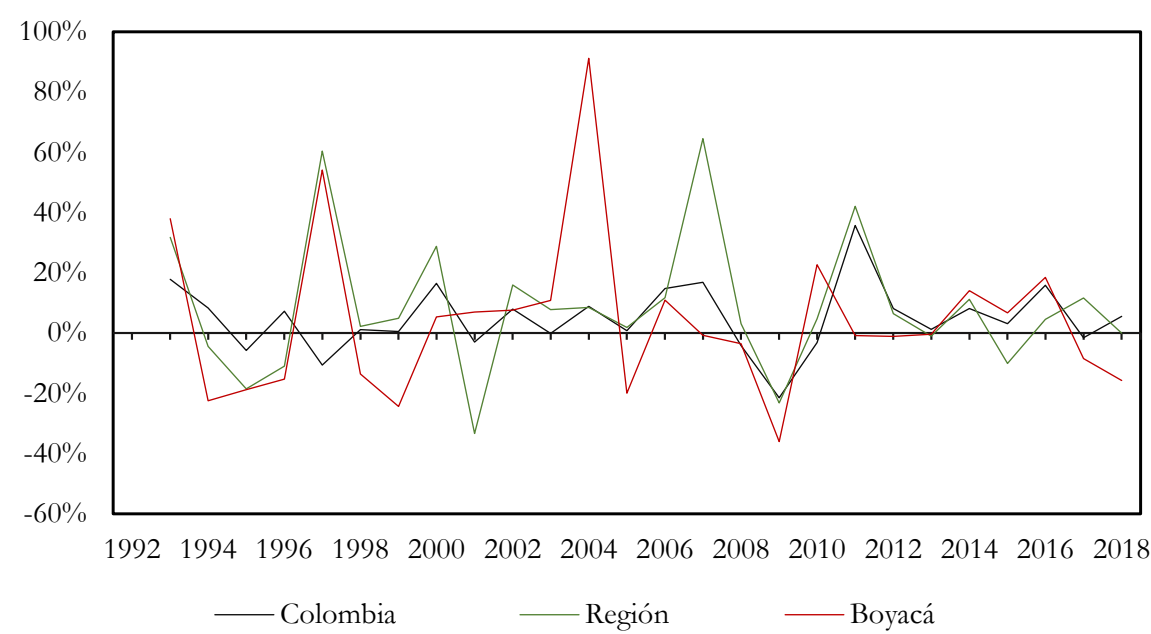




\section{(c) Gasto en capital}

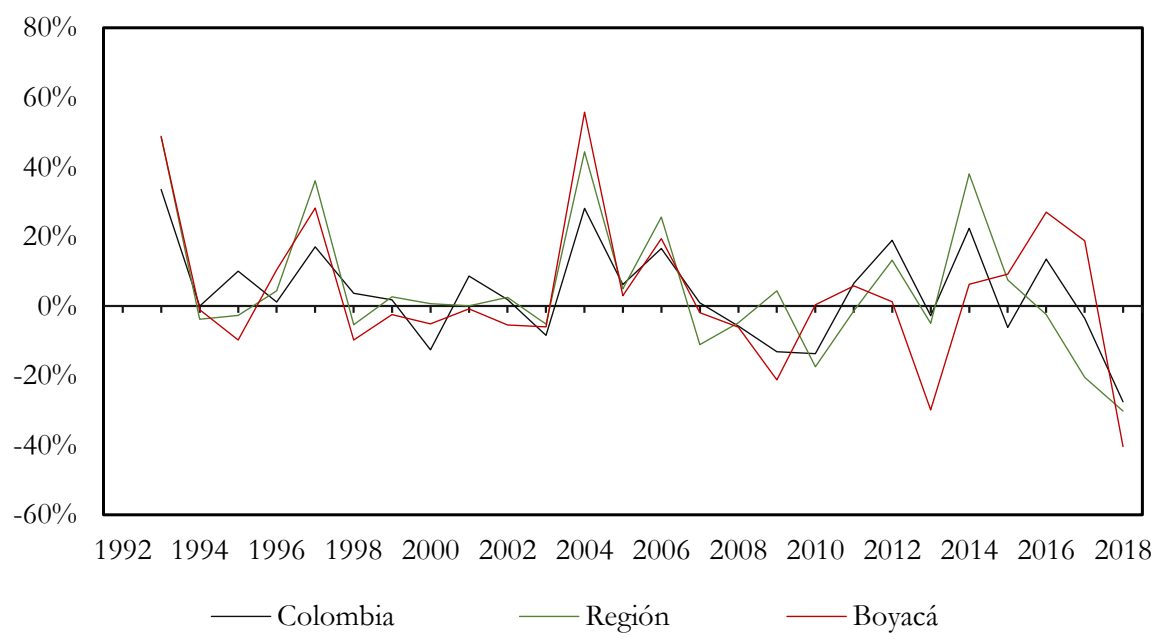

(d) Gasto en energía

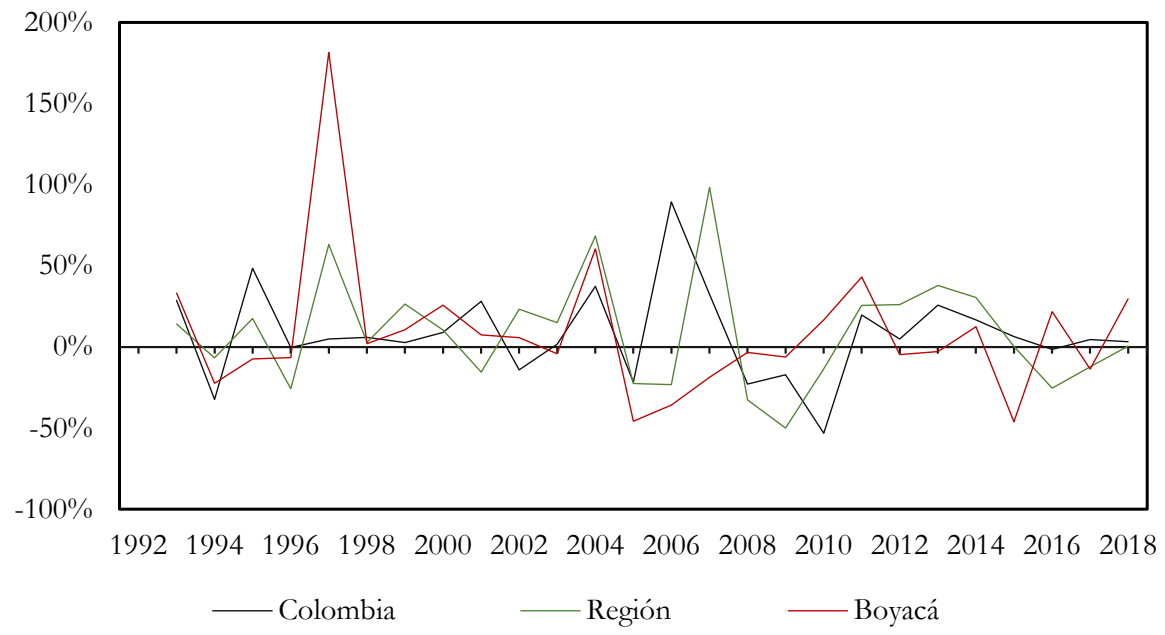


Casas Herrera y Gutiérrez Ayala: Análisis territorial de las elasticidades de sustitución...

(e) Gasto en otros factores de producción

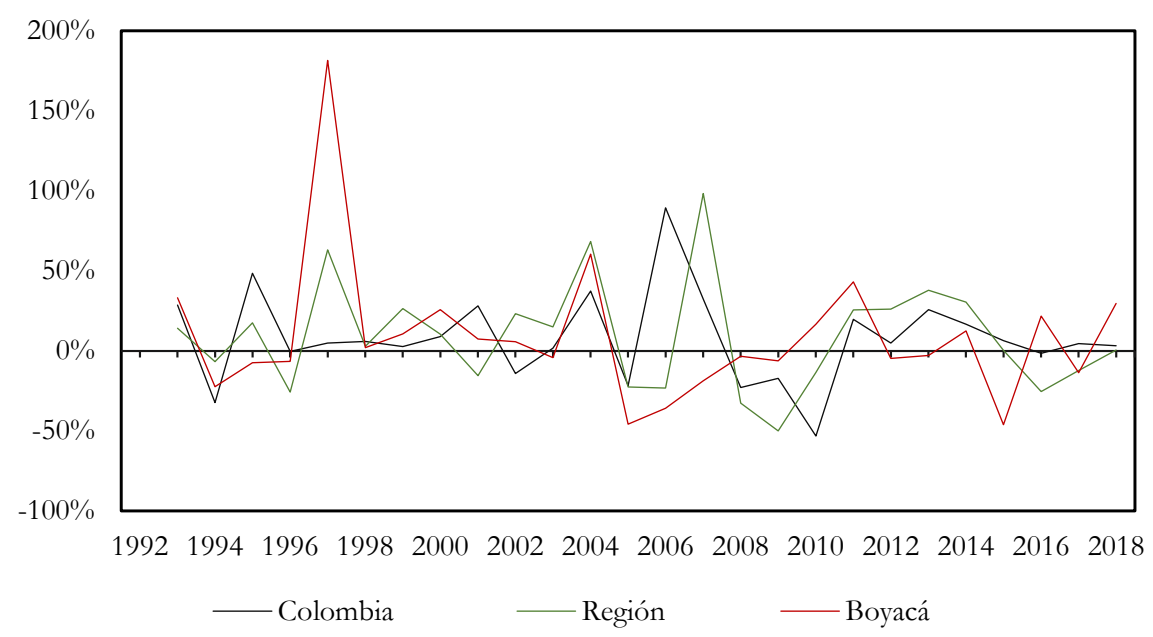

Fuente: elaboración propia con base en datos del DANE (2019).

El gasto en capital presenta un comportamiento muy similar en los tres niveles territoriales (panel c). De hecho, la tasa de variación anual promedio en la industria colombiana fue de $3,73 \%$, valor que casi coincide con el de Boyacá $(3,61 \%)$. Esto muestra que el comportamiento del índice de precios del capital es cercano en el total de la industria nacional.

Similar análisis aplica para el gasto en energía eléctrica (panel d), el cual a partir del 2004 tiende a una estabilización para la industria colombiana, regional y boyacense. Finalmente, el gasto en otros factores de producción (panel e) presenta las variaciones más altas en los tres niveles territoriales examinados. La tasa media de variación en la industria nacional fue de $7.98 \%$ y para la región y Boyacá se obtuvo una variación media de $8.97 \%$.

En general, las empresas de Boyacá experimentaron tasas de crecimiento del gasto en insumos más bajas que el promedio regional y nacional, por lo que se plantea la existencia de posibles diferencias entre las relaciones entre demanda y precio de los factores a nivel empresarial. En este sentido, la Tabla 1 muestra la participación promedio del precio de los factores en el costo total en el periodo de referencia. 
Tabla 1. Participación promedio del precio de los factores en el costo total de producción (1992-2018)

\begin{tabular}{lccc}
\hline Factor & Colombia & Región & Boyacá \\
\hline Trabajo & $15,33 \%$ & $12,72 \%$ & $20,36 \%$ \\
Materias primas & $61,82 \%$ & $66,08 \%$ & $43,08 \%$ \\
Capital & $10,18 \%$ & $9,15 \%$ & $16,16 \%$ \\
Energía & $2,42 \%$ & $2,44 \%$ & $8,48 \%$ \\
Otros factores & $10,25 \%$ & $9,61 \%$ & $11,92 \%$ \\
\hline
\end{tabular}

Fuente: elaboración propia con base en datos del DANE (2019).

Los datos de la Tabla 1 muestran que la industria manufacturera de Colombia (nacional) contó con un uso intensivo de materias primas y trabajo (77\%). En el caso de la región la participación de estos factores fue del 79\% y para la industria de Boyacá fue del $63 \%$. Aunque vale destacar que hubo un mayor costo en mano de obra (20\%) y uno menor en materias primas $(43 \%)$, en comparación con las otras dos unidades de análisis.

El costo de capital (arrendamientos, uso de maquinaria e intereses financieros) representó, en promedio, para la industria manufacturera colombiana, de la región y de Boyacá un 10,2\%, 9,15\% y 16,16\% respectivamente. Esto permite inferir que las empresas manufactureras del departamento son más intensivas en el uso de capital que sus similares a nivel regional y nacional.

La participación relativa del costo en energía eléctrica a nivel nacional y de la región fue de $2,4 \%$, mientras que para la industria manufacturera del departamento, fue de $8,48 \%$. De lo anterior se deduce que existe una disparidad en el uso de este factor de producción. Esta disparidad se explica, por una parte, por la diferencia del uso de kilowatts en el proceso de producción; y por otra, por la diferencia que puede existir en el precio por unidad energética consumida.

El costo por otros factores de producción tuvo una participación similar en promedio para Colombia (10,25\%), la región $(9,61 \%)$ y Boyacá $(11,92 \%)$. 
Casas Herrera y Gutiérrez Ayala: Análisis territorial de las elasticidades de sustitución...

Esto permite centrarse en las diferencias existentes entre el uso de los factores: trabajo, materias primas, capital y energía.

Como conclusión preliminar, se puede observar que las empresas manufactureras de Boyacá tienen una mayor participación del costo de mano de obra y capital, frente a las empresas de la región y del país. Esta diferencia puede explicarse por dos razones, por un lado, por la diferencia en los índices de precios de los factores; y por otro, por la diferencia en la cantidad de trabajadores y maquinaria empleados en el proceso de producción.

Con el objetivo de identificar diferencias en la relación entre los factores de producción en la industria manufacturera nacional, regional y departamental, se procede a estimar las curvas de demanda de los factores de producción, junto con sus elasticidades precio y elasticidades cruzadas Allen-Uzawa.

\section{Estimación y discusión de los resultados}

El sistema de ecuaciones (representado en 9) fue estimado, para los tres niveles territoriales, usando el método de ecuaciones aparentemente no relacionadas (SURE). Ya que, como se mencionó, este permite la obtención de estimadores más eficientes frente al uso de MCO. La Tabla 2 muestra las estimaciones de las curvas de demanda de los factores de producción para los tres niveles. En la primera columna están los parámetros a estimar. En las columnas dos, tres y cuatro se presentan los resultados correspondientes a Colombia, la región y Boyacá, respectivamente.

Tabla 2. Resultados de la estimación de la curva de demanda de los factores de producción

\begin{tabular}{cccc}
\hline & Colombia & Región & Boyacá \\
\hline$\alpha_{L}$ & $0,121^{* * *}$ & $0,091^{* * *}$ & $0,197^{* * *}$ \\
& $(0,006)$ & $(0,02)$ & $(0,033)$ \\
$\gamma_{L L}$ & $0,128^{* * *}$ & $0,111^{* * *}$ & $0,155^{* * *}$ \\
& $(0,001)$ & $(0,003)$ & $(0,004)$ \\
$\gamma_{L M}$ & $-0,093^{* * *}$ & $-0,080^{* * *}$ & $-0,081^{* * *}$ \\
& $(0,001)$ & $(0,002)$ & $(0,002)$ \\
\hline & & & Continúa
\end{tabular}


Tabla 2. Continúa

\begin{tabular}{|c|c|c|c|}
\hline & Colombia & Región & Boyacá \\
\hline$\gamma_{L K}$ & $\begin{array}{c}-0,014^{* * *} \\
(0,001)\end{array}$ & $\begin{array}{c}-0,009^{* * *} \\
(0,002)\end{array}$ & $\begin{array}{c}-0,036^{* * *} \\
(0,002)\end{array}$ \\
\hline$\gamma_{L E}$ & $\begin{array}{c}-0,001^{* * *} \\
(0,0005)\end{array}$ & $\begin{array}{c}-0,008^{* * *} \\
(0,0009)\end{array}$ & $\begin{array}{c}-0,017^{* * *} \\
(0,002)\end{array}$ \\
\hline$\gamma_{L Y}$ & $\begin{array}{c}0,0001 \\
(0,0003)\end{array}$ & $\begin{array}{c}-0,00009 \\
(0,001)\end{array}$ & $\begin{array}{l}-0,001 \\
(0,001)\end{array}$ \\
\hline$\alpha_{M}$ & $\begin{array}{c}0,689^{* * *} \\
(0,009)\end{array}$ & $\begin{array}{c}0,805^{* * *} \\
(0,016)\end{array}$ & $\begin{array}{c}0,392^{* * *} \\
(0,022)\end{array}$ \\
\hline$\gamma_{M M}$ & $\begin{array}{c}0,235^{* * *} \\
(0,001)\end{array}$ & $\begin{array}{c}0,218^{* * *} \\
(0,002)\end{array}$ & $\begin{array}{c}0,242^{* * *} \\
(0,003)\end{array}$ \\
\hline$\gamma_{M K}$ & $\begin{array}{c}-0,059^{* * *} \\
(0,001)\end{array}$ & $\begin{array}{c}-0,056^{* * *} \\
(0,001)\end{array}$ & $\begin{array}{c}-0,070^{* * *} \\
(0,002)\end{array}$ \\
\hline$\gamma_{M E}$ & $\begin{array}{c}-0,014^{* * *} \\
(0,0003)\end{array}$ & $\begin{array}{c}-0,016^{* * *} \\
(0,0005)\end{array}$ & $\begin{array}{c}-0,040^{* * *} \\
(0,002)\end{array}$ \\
\hline$\gamma_{M Y}$ & $\begin{array}{c}0,0001 \\
(0,0005)\end{array}$ & $\begin{array}{c}-0,001 \\
(0,0009)\end{array}$ & $\begin{array}{c}0,004^{* * *} \\
(0,001)\end{array}$ \\
\hline$\alpha_{K}$ & $\begin{array}{c}0,060^{* * *} \\
(0,007)\end{array}$ & $\begin{array}{l}0,029^{* *} \\
(0,014)\end{array}$ & $\begin{array}{c}0,150^{* * *} \\
(0,019)\end{array}$ \\
\hline$\gamma_{K K}$ & $\begin{array}{c}0,086^{* * *} \\
(0,002)\end{array}$ & $\begin{array}{c}0,078^{* * *} \\
(0,003)\end{array}$ & $\begin{array}{c}0,138^{* * *} \\
(0,002)\end{array}$ \\
\hline$\gamma_{K E}$ & $\begin{array}{c}-0,002^{* * *} \\
(0,0004)\end{array}$ & $\begin{array}{c}-0,002^{* * *} \\
(0,0007)\end{array}$ & $\begin{array}{c}-0,009^{* * *} \\
(0,001)\end{array}$ \\
\hline$\gamma_{K Y}$ & $\begin{array}{c}-0,00001 \\
(0,0004)\end{array}$ & $\begin{array}{c}0,0004 \\
(0,0008)\end{array}$ & $\begin{array}{l}-0,001 \\
(0,001)\end{array}$ \\
\hline$\alpha_{E}$ & $\begin{array}{c}0,022^{* * *} \\
(0,001)\end{array}$ & $\begin{array}{c}0,036^{* * *} \\
(0,004)\end{array}$ & $\begin{array}{c}0,123^{* * *} \\
(0,019)\end{array}$ \\
\hline$\gamma_{E E}$ & $\begin{array}{l}0,020^{* * *} \\
(0,0003)\end{array}$ & $\begin{array}{l}0,028^{* * *} \\
(0,0006)\end{array}$ & $\begin{array}{c}0,079^{* * *} \\
(0,002)\end{array}$ \\
\hline$\gamma_{E Y}$ & $\begin{array}{c}-0,00004 \\
(0,0001)\end{array}$ & $\begin{array}{c}-0,009^{* * *} \\
(0,0002)\end{array}$ & $\begin{array}{c}-0,0003 \\
(0,001)\end{array}$ \\
\hline $\mathbf{R}^{2}(\mathrm{~L})$ & 0,99 & 0,99 & 0,99 \\
\hline $\mathrm{R}^{2}(\mathbf{M})$ & 0,99 & 0,99 & 0,99 \\
\hline $\mathbf{R}^{2}(\mathbf{K})$ & 0,98 & 0,98 & 0,98 \\
\hline $\mathbf{R}^{2}(\mathrm{E})$ & 0,99 & 0,98 & 0,97 \\
\hline
\end{tabular}


Casas Herrera y Gutiérrez Ayala: Análisis territorial de las elasticidades de sustitución...

Fuente: elaboración propia con datos del DANE (2019). Estimación realizada en Eviews 10.

Como se puede observar, se encuentra significancia estadística para la mayoría de estimadores, exceptuando aquellos que relacionan la demanda de cada factor con el nivel de producción de la industria. Esto significa que, aunque sí existe una relación directa entre el uso de factores y la producción, el mercado de insumos y mano de obra es independiente del nivel del producto.

Vale la pena señalar que al usar la metodología SURE, el $\mathrm{R}^{2}$ se estima de manera diferente frente a MCO, ya que según Cabezas (1997, p. 521) “el $\mathrm{R}^{2}$ tradicional no es una medida adecuada para una ecuación individual en un sistema de ecuaciones, pues se puede obtener un valor negativo para una ecuación en particular". En esta metodología, la bondad de ajuste se mide como el cuadrado del coeficiente de correlación entre los valores observados y estimados de cada una de las variables endógenas. También se observa, en la Tabla 2, que los valores obtenidos para el $\mathrm{R}^{2}$ son elevados por lo que hay un alto grado de significancia global.

Ahora bien, una vez calculados los estimadores que muestran la relación entre la demanda y los precios de los factores de producción, lo más lógico es que se proceda a analizar los valores obtenidos. Sin embargo, hay que aclarar que los estimadores calculados mediante SURE no cuentan con un análisis económico que pueda presentarse, sino que estos son la base para la verificación de los supuestos de homogeneidad, simetría y el cálculo de las elasticidades parciales (Allen-Uzawa) y las elasticidades propias de la demanda de factores de producción, fin para el cual se hace uso de las ecuaciones $10 \mathrm{y}$ 11 , respectivamente. Estas elasticidades se calculan y analizan a continuación (ver Tabla 3).

Con el objetivo de ser explicativos, pero no reiterativos, se interpretará únicamente la elasticidad parcial trabajo-capital (ya que esta relación es la más estudiada) y las elasticidades propias con signo positivo (por representar un alto grado de dependencia por parte de la industria hacia la demanda de un factor en específico). Esto porque el ejercicio explicativo se haría muy extenso, si se hiciera para las 14 elasticidades. No obstante, se invita al lector a realizar los análisis que sean de su interés siguiendo la lógica interpretativa que se deja en los siguientes cuatro párrafos. 
Como se mencionó en la sección de antecedentes, las elasticidades de sustitución entre factores de producción representan el cambio de la demanda de un factor $i$ ante un cambio en el precio de un factor $j$. Esta relación permite analizar, por un lado, el sentido del cambio; y por otro, su magnitud. En cuanto al sentido, este se evalúa mediante el signo estimado de la elasticidad que determina las relaciones de sustitución (signo positivo representa que un aumento del precio de $j$ genera un aumento de la demanda de i) y complementariedad (signo negativo representa que un aumento del precio de $j$ genera una reducción de la demanda de $i$ ). Respecto de la magnitud del cambio, esta se determina por el valor absoluto estimado para la elasticidad y permite inferir el uso intensivo de un factor determinado, entre más grande sea representará que para una industria es más fácil cambiar un factor por otro.

Tabla 3. Elasticidades precio y elasticidades parciales (Allen-Uzawa) de la demanda de insumos

\begin{tabular}{lccc}
\hline Elasticidades & Colombia & Región & Boyacá \\
\hline$\sigma_{L L}$ & $-0,053$ & 0,056 & $-0,171$ \\
$\sigma_{L M}$ & 0,008 & 0,037 & 0,072 \\
$\sigma_{L K}$ & 0,086 & 0,189 & $-0,121$ \\
$\sigma_{L E}$ & 0,598 & $-1,581$ & $-0,006$ \\
$\sigma_{L O}$ & $-0,196$ & $-0,107$ & 0,199 \\
$\sigma_{M M}$ & $-0,0007$ & $-0,012$ & $-0,015$ \\
$\sigma_{M K}$ & 0,051 & 0,059 & $-0,018$ \\
$\sigma_{M E}$ & 0,017 & $-0,008$ & $-0,11$ \\
$\sigma_{M O}$ & $-0,062$ & $-0,018$ & 0,033 \\
$\sigma_{K K}$ & $-0,453$ & $-0,514$ & 0,098 \\
$\sigma_{K E}$ & 0,17 & $-0,24$ & 0,308 \\
$\sigma_{K O}$ & $-0,03$ & $-0,111$ & $-0,077$ \\
$\sigma_{E E}$ & $-4,54$ & 8,179 & 0,223 \\
$\sigma_{E O}$ & $-0,081$ & 0,703 & $-0,13$ \\
\hline
\end{tabular}

Fuente: elaboración propia. 
Casas Herrera y Gutiérrez Ayala: Análisis territorial de las elasticidades de sustitución...

En el caso de la elasticidad parcial trabajo-capital $\sigma_{L K}$ (ver fila 3 de la Tabla 3), la industria manufacturera nacional mostró en promedio para el periodo 1992-2018, un efecto positivo de 0,09\%, caracterizando una relación de sustitución entre estos factores, en la que el aumento de un $1 \%$ del precio del capital causó un crecimiento en la demanda de trabajo de 0,09\%. En el caso de la región se encontró, de igual forma, una relación de sustitución; sin embargo, esta fue más sensible ${ }^{15}(0,18 \%)$. Para la industria manufacturera de Boyacá la elasticidad fue negativa $(-0,12 \%)$, esto significa que la relación trabajo-capital fue complementaria; es decir, en el caso de las empresas boyacenses la demanda de mano de obra se redujo cuando el precio del capital aumentó.

Los resultados muestran que en cuanto a la relación trabajo-capital, la industria nacional cuenta con una magnitud de $0,09 \%$, la cual equivale a la mitad de la magnitud estimada para la región $(0,18 \%)$. Esto permite inferir que la industria colombiana tiene un uso más intensivo de capital que la región, ya que le es más difícil sustituir trabajo por capital ${ }^{16}$. Este resultado se comprueba al observar la elasticidad propia del capital $\sigma_{K K}$ (fila 10 de la Tabla 3) cuya magnitud es mayor en la región (-0,51\%) frente a la de la industria nacional $(-0,45 \%)$, lo que representa que en el nivel regional hay una mayor sensibilidad por parte del factor capital y, por ende, es más fácil reemplazarlo por otros factores. En el caso de Boyacá se comprueba que, al ser un departamento caracterizado por tener una industria metalera y de producción de alimentos, hay una tendencia a mantener un alto uso tanto de mano de obra como de capital, esto lleva a tener una relación de complementariedad (de ahí el signo negativo).

En cuanto a las elasticidades propias, estas muestran el efecto de un aumento en el precio de un factor de producción sobre la variación de su propia demanda. Estas permiten identificar el grado de dependencia que tiene una industria frente al uso de un factor en específico, ya que, aunque lo normal es encontrar elasticidades propias negativas ${ }^{17}$, es posible obtener elasticidades

15 En términos técnicos se plantearía que es más elástica.

16 A menor magnitud, menor grado de sustitución y por ende menor efecto entre factores.

17 Si la elasticidad propia es negativa, se entiende, que un aumento del precio del insumo lleva a una reducción de su demanda. 
positivas. Las cuales significan que un aumento del precio del factor causa un aumento de su demanda y reflejan que la industria depende a tal nivel de su uso que no importa si el precio aumenta, se seguirá adquiriendo. Para este caso, se encontraron elasticidades propias positivas para la industria regional. Es decir, ante una variación del precio propio de un $1 \%$ se generó un aumento de la demanda de la mano de obra y la energía en $0,05 \%$ y $8,1 \%$ respectivamente. Para la industria de Boyacá, los factores que aumentaron su demanda, ante incrementos de su precio, fueron el capital $(0,1 \%)$ y la energía $(0,22 \%)$.

\section{Conclusiones}

El objetivo del artículo es estimar las elasticidades propias y cruzadas de demanda de los factores de producción para el periodo 1992-2018 en la industria manufacturera colombiana, regional y del departamento de Boyacá, con el propósito de identificar diferencias en los distintos niveles territoriales de la industria. Para este fin se tomaron datos de la EAM referentes al gasto empresarial en los factores trabajo, materias primas, capital, energía y otros gastos de producción. Además, se construyó un sistema de ecuaciones que recoge las funciones de demanda no restringida de los factores mencionados. Esto a partir de una función de costos translogarítmica. El sistema 9 fue calculado mediante la metodología SURE, la cual permite corregir los problemas de colinealidad que caracterizan a sistemas que comparten variables exógenas como en este caso.

Los resultados muestran la existencia de diferencias a nivel territorial en las elasticidades calculadas. Por ejemplo, la elasticidad cruzada trabajo-capital mostró una relación de sustitución en los niveles nacional y regional $(0,09 \%$ y $0,18 \%$ respectivamente), contrario al caso de la industria manufacturera de Boyacá, donde se encontró una relación complementaria entre factores $(-0,12 \%)$. Esto quiere decir que mientras que a nivel nacional y regional los aumentos de la demanda de capital causaron reducciones en el gasto en mano de obra, a nivel departamental estos factores aumentaron su demanda de forma conjunta. Igual análisis aplica para la elasticidad de las materias primas-capital, que mostró que la industria del departamento tuvo patrones de demanda diferentes a la nación y la región. 
Casas Herrera y Gutiérrez Ayala: Análisis territorial de las elasticidades de sustitución...

Las elasticidades propias calculadas para la industria nacional fueron negativas, encontrando una relación lógica en la que el aumento del precio de un factor genera reducciones de su demanda. Sin embargo, cuando se analizan a nivel regional y departamental, se encontró un alto grado de dependencia de los factores mano de obra, capital y energía.

Al comparar los resultados obtenidos con los antecedentes presentados en otros documentos, se encuentra que la industria manufacturera colombiana, al igual que la industria mexicana, tienen una relación sustitutiva entre trabajo y capital, tal como lo muestran Salgado y Bernal (2010). Así mismo, el resultado coincide con el encontrado por Cabezas (1997) para actividades industriales como la producción de joyería, juguetes y artículos de oficina en Perú. En el caso de la industria manufacturera de Boyacá, la relación de complementariedad entre estos factores coincidió con los resultados de Cabezas (1997) para actividades como producción de alimentos, textiles, productos de madera, papel, maquinaria y minerales no metálicos.

Por último, Ramírez Hassan (2008) encontró para la industria colombiana una relación complementaria entre capital y trabajo calificado, así como una relación sustitutiva entre capital y trabajo no calificado, siendo esta última de mayor magnitud. Por lo que se puede inferir una relación de sustitución entre el trabajo en general y el capital, lo que coincide con los resultados obtenidos en este artículo. Finalmente, en el caso de las elasticidades propias, se obtuvo una coherencia en los signos obtenidos.

El análisis realizado permite identificar diferencias sustanciales en el comportamiento de la industria manufacturera en tres niveles territoriales. Las estimaciones realizadas muestran que las dinámicas de demanda de factores de producción dependen del territorio en el que se encuentra la industria, y que hay comportamientos distintivos en cuanto a los tipos de industria, los salarios, precio del capital, materias primas e incluso energía eléctrica. Esto lleva a redefinir el alcance de políticas y estrategias pensadas desde un nivel macro, considerando los posibles efectos contrarios que pueden presentarse en distintos niveles del territorio nacional. Es posible que falte analizar otras relaciones o características de la industria manufacturera en Colombia, pero esto será sujeto de otros análisis o investigaciones. 


\section{Referencias}

Aigner, D., Lovell, K. \& Schmidt, P. (1977). Formulation and Estimation of Stochastic Frontier Production Function Models. Journal of Econometrics, 6, 21-37. https://doi.org/10.1016/0304-4076(77)90052-5

Allen, R. G. D. (1938). Mathematical Analysis for Economists. Londres: MacMillan.

Álvarez, A., Arias, C. \& Orea, L. (2003). Introducción al análisis empirico de la producción. Universidad de Oviedo.

Álvarez Mendoza, A. T. \& Defaz Novillo, S. S. (2019). Relación entre la capacitación del personal y productividad en la industria manufacturera de productos alimenticios del cantón Manta-Ecuador. Universidad Católica Santiago de Guayaquil.

Amézquita, C. (2008). Manufacturing Sector in Colombia (1995-2005). Revista de la Facultad de Ciencias Económicas, XVI (2), 61-78. Recuperado a partir de https://revistas.unimilitar.edu.co/index.php /rfce/article/view/4467

Andrade, J., Gaspar, D. \& Bittencourt, A. (2014). América Latina: productividad total de los factores y su descomposición. Revista de la Cepal, 114, 53-69. https://doi.org/10.18356/0e0eafe2-es

Ávila, C., Camargo, W. \& Marques, N. (2019). Localización y especialización productiva: el caso de la región amazorinoquia de Colombia. En F. dos Santos (Ed.), Geografia no Século XXI. https://doi.org/10.36229/978-8 $5-7042-140-1$

Baltagi, B. (2006). Seemingly Unrelated Regressions. Springer. https://doi.org/ 10.1007/978-3-642-20059-5

Berndt, E. R. \& Christensen, L. R. (1973). The translog function and the substitution of equipment, structures, and labor in U.S. manufacturing 1929-68. Journal of Econometrics, 1(1), 81-113. https://doi.org/10.101 6/0304-4076(73)90007-9 
Casas Herrera y Gutiérrez Ayala: Análisis territorial de las elasticidades de sustitución...

Berndt, E. \& Wood, D. (1987). Technology Prices and the Derived Demand for Energy. The Review of Economics and Statistics, 57(3), 259-268. https: //doi.org/10.2307/1923910

Cabezas, L. (1997). Sustitución entre factores de producción y cambio técnico en la industria manufacturera peruana. El Trimestre Económico, 64(256), 513-530. http://aleph.academica.mx/jspui/bitstream/56789/5847/1/ DOCT2065102_ARTICULO_3.PDF

Carro, R. \& González, D. (2019). Productividad y Competitividad. Universidad Nacional de Mar de Plata. http://nulan.mdp.edu.ar/160 7/1/02_productividad_competitividad.pdf

Cepas, S. \& Dios, R. (1999). Análisis de la función de producción agraria para distintos niveles de agregación. Estudios de Economía aplicada, 12, 17-33. https://www.researchgate.net/publication/28088619_Analisis_d e_la_Funcion_de_Produccion_Agraria_para_distintos_niveles_de_Agr egacion

Christensen, L. R., Jorgenson, D. W. \& Lau, L. J. (1973). Transcendental Logarithmic Production Frontiers. The Review of Economics and Statistics, 55(1), 28-45. http://www.jstor.org/stable/1927992

Clemson University (2005). Transcendental Logarithmic Cost Function. En Encyclopedia of Statistical Sciences. https://doi.org/10.1002/0471667196 .ess2747.pub2

Cobb, C. \& Douglas, P. (1928). A Theory of Production. The American Economic Review, 18(1). http://www2.econ.iastate.edu/classes/econ 521/Orazem/Papers/cobb-douglas.pdf

Departamento Administrativo Nacional de Estadística (DANE) (2019). Metodología General Encuesta Anual Manufacturera-EAM. https://www. dane.gov.co/index.php/estadisticas-por-tema/industria/encuesta-anual -manufacturera-enam

Diewert, W. E. (1971). An Application of the Shephard Duality Theorem: A Generalized Leontief Production Function. Journal of Political Economy, 79(3), 481-507. https://doi.org/10.1086/259764 
Geweke, J. (2005). Contemporary Bayesian Econometrics and Statistics. En Contemporary Bayesian Econometrics and Statistics. https://doi.org/10.1 002/0471744735

Gujarati, D. \& Porter, D. (2010). Econometría (5ta. ed.). McGraw-Hill.

Hisnanick, J. J. \& Kyer, B. L. (1995). Assessing a disaggregated energy input. Using confidence intervals around translog elasticity estimates. Energy Economics, 17(2), 125-132. https://doi.org/10.1016/0140-9883(95)0 0008-I

Ibarra, J. \& García, F. (2013). Elasticidades de sustitución y separabilidad de los factores productivos de la industria. Estudios Fronterizos, 14, 155-185. https://doi.org/10.21670/ref.2013.28.a07

Ibarra, J. \& García, F. (2016). Las demandas de factores productivos en la industria maquiladora. Estudios Económicos, 31, 265-303. https://doi.or g/10.24201/ee.v31i2.18

Kuan, C., Hongchang, W., Yuxin, Z., Jefferson, G. H. \& Rawski, T. G. (1988). Productivity change in Chinese industry: 1953-1985. Journal of Comparative Economics, 12(4), 570-591. https://doi.org/10.1016/0147 -5967(88)90104-7

Le, P. V. (2019). Energy demand and factor substitution in Vietnam: evidence from two recent enterprise surveys. Journal of Economic Structures, 8(35). https://doi.org/10.1186/s40008-019-0168-9

Looi Kee, H., Nicita, A. \& Olarreaga, M. (2008). Import demand elasticities and trade distortions. The Review of Economics and Statistics, 90(4), 666682. https://doi.org/10.1162/rest.90.4.666

Mundlak, Y. (1968). Elasticities of Substitution and the Theory of Derived Demand. The Review of Economic Studies, 35(2), 225-236. http://www. jstor.org/stable/2296550

Ramírez Hassan, A. (2008). Una función de producción agregada para la economía colombiana: características e interacción entre el trabajo calificado, el trabajo no calificado y el capital, 1994-2005. Ecos de Economia, 26, 41-70. https://doi.org/10.2139/ssrn.2476356 
Casas Herrera y Gutiérrez Ayala: Análisis territorial de las elasticidades de sustitución...

Rossi, P. E. (1985). Comparison of alternative functional forms in production. Journal of Econometrics, 30(1-2), 345-361. https://doi.org/10.1016/03 04-4076(85)90145-9

Saal, D. S. \& Parker, D. (2000). The impact of privatization and regulation on the water and sewerage industry in England and Wales: A translog cost function model. Managerial and Decision Economics, 21(6), 253-268. https://doi.org/10.1002/mde.988

Salgado, H. \& Bernal, L. (2010). Funciones de costos translogarítmicas: Una aplicación para el sector manufacturero mexicano. Trimestre Economico, 77(307), 683-717. https://doi.org/10.20430/ete.v77i307.450

Uzawa, H. (1962). Production Functions Elasticities of Substitution. The Review of Economic Studies, 29(4), 291-299. https://doi.org/10.230 $7 / 2296305$

Vidal, N. (2020). Análisis de la participación del sector manufacturero en el Ecuador en la producción mundial: una perspectiva desde la productividad real de la Economía. (2005-2018) (Universidad Católica de Santiago de Guayaquil). http://repositorio.ucsg.edu.ec/handle/3317/14486

Zellner, A. (1962). An Efficient Method of Estimating Seemingly Unrelated Regressions and Tests for Aggregation Bias. Journal of the American Statistical Association, 57(298), 348-368. https://doi.org/10.2307/22 81644

Zha, D. \& Zhou, D. (2014). The elasticity of substitution and the way of nesting CES production function with emphasis on energy input. Applied Energy, 130, 793-798. https://doi.org/10.1016/j.apenergy.2 014.01 .093 